\title{
AVALIAÇÃO DO PROCESSO DE DESIDRATAÇÃO OSMÓTICA DE PIMENTA MALAGUETA (Capsicum frutens), VARIEDADE MALAGUETINHA.
}

Evaluation of osmotic dehydration process of chilli pepper (Capsicum frutens) $\mathrm{cv}$ malaguetinha

Evaluación del proceso de desidratación osmótica de pimienta malagueta (Capsicum

Frutens) variedad malaguetinha.

\section{Denise Gomes Alves ${ }^{* 1}$, Camila Noleto Abreu ${ }^{1}$, Hermanny Matos Silva Sousa ${ }^{1}$, Cecília Marques Tenório Pereira ${ }^{1}$}

${ }^{1}$ Curso de Nutrição, Universidade Federal do Tocantins, Palmas, Tocantins, Brasil.

*Correspondência: Curso de Nutrição, Universidade Federal do Tocantins, Av. NS 15, 109 Norte, Palmas, Tocantins, Brasil.CEP:77.010-090.e-mail denise@uft.edu.br

\section{RESUMO}

Apreciada há milhares de anos, a pimenta malagueta (Capsicum frutescens) está presente na mesa da população espalhada por todo o mundo, sendo utilizada na maioria das vezes como especiaria. Em consequência desta procura, a área de cultivo de pimentas nos vários estados brasileiros expandiu, com o intuito de atender tanto o mercado interno quanto o externo. Elas abastecem a agroindústria, são consumidas in natura e processadas para serem utilizadas em várias linhas de produtos. Este trabalho teve como objetivo a avaliação do processo de desidratação osmótica de pimenta malagueta (Capsicum frutens). Não houve diferenças significativas entre as soluções desidratantes tanto para a perda de água (PA) como para o ganho de sólidos (GS).

Palavras-chave: desidratação, pimenta malagueta, processamento.

\section{ABSTRACT}

Chilli pepper (Capsicum frutescens) is appreciated for thousands of years as spice and it is on population's table all over the world. To attend consumers' demand and local markets as well as foreign market this crop has expanded in several Brazilians states. They provide material for food and agricultural industries and can be consumed in natura and processed in other products lines. The objective of this work was to optimize osmotic dehydration process of chilli pepper (Capsicum frutescens) using dehydrating solutions with sugar/cinnamon tea and sugar/distilled water to observe water loss (WL) and solid gain $(S G)$. There were no significant differences between the dehydrating solutions for both water loss (WL) and solids gain (SG).

Keywords: dehydration, chilli pepper, processing.

\section{RESUMEN}

Apreciada hace miles de años, la pimienta malagueta (Capsicum frutescens) está presente en la mesa de la población esparcida por todo el mundo, siendo utilizada en la mayoría de las veces como especia. En consecuencia de esta demanda, el área de cultivo de pimientos en los diversos estados brasileños se expandió, con el objetivo de atender tanto el mercado interno como el externo. Ellas abastecen a la agroindustria, son consumidas in natura y procesadas para ser utilizadas en varias líneas de productos. Este trabajo tuvo como objetivo la optimización del proceso de deshidratación osmótica de pimienta malagueta (Capsicum frutens). No hubo diferencias significativas entre las soluciones deshidratantes tanto para la pérdida de agua (PA) como para la ganancia de sólidos (GS).

Descriptores: deshidratación, pimienta malagueta, procesamiento 


\section{INTRODUÇÃO}

A preservação de alimentos através de secagem é um processo já conhecido desde as épocas mais remotas. Os povos das civilizações antigas descobriram que o sal poderia funcionar como agente desidratante, conservando assim o alimento por muito tempo.

Utilizar agentes desidratantes para retirar água de alimentos é um processo conhecido como desidratação osmótica. Esse processo não retira toda a umidade, contudo pode ser utilizado como um prétratamento antes da secagem convencional, produzindo alimentos de umidade intermediária. Tem como finalidade a diminuição da perecibilidade, preservando as características naturais dos produtos. A utilização de sal ou açúcar como agente desidratante é muito comum no processo de desidratação osmótica. Normalmente para as frutas o açúcar é o agente mais utilizado. Soluções desidratantes mais concentradas produzem maior perda de água, o que pode possibilitar o aumento da incorporação de sólidos. Entre as técnicas de conservação de alimentos, a desidratação osmótica tem sido muito estudada com o objetivo de avaliar que variáveis são mais importantes dentro do processo. $\mathrm{O}$ aumento da temperatura de osmose influencia significativamente a quantidade de água durante todo o processo de desidratação como, por exemplo, nas goiabas, porém não influencia a quantidade de sacarose final do produto (CASTRO et. al., 2014).

A literatura mostra que na avaliação do processo de desidratação osmótica é complexo incluir todos os fatores envolvidos na atividade, o que dificulta a otimização do processo a ser utilizado em diferentes produtos. Até mesmo quando o processo é aplicado sobre um mesmo produto, há a possibilidade de se chegar a conclusões que podem divergir entre si. Como exemplo, as conclusões de Castro et. al. (2014) foram divergentes dos resultados obtidos por SanjinezArgadoña et. al. (2004) no estudo da influência das variáveis do processo de desidratação osmótica de pedaços de goiaba.

Segundo Bianco (2000), a termogênese pode ser considerada um processo onde os alimentos liberam energia química durante a oxidação de gorduras e açucares, podendo este procedimento ser caracterizado de duas formas inerentes: termogênese obrigatória e a termogênese facultativa. A primeira está relacionada, considerando o ponto de vista fisiológico, aos métodos essenciais aos mecanismos de troca e armazenamento de energia do corpo. A segunda é variável e pode derivar de estímulos desencadeadores, como por exemplo, quando algo é exposto ao frio e busca equilibrar a temperatura produzindo calor. Além do frio, existem alimentos ou substâncias que quando ingeridas ou quando entram em contato com alguma parte do corpo, alteram os processos metabólicos do organismo, fazendo com que ele trabalhe com maior intensidade. Esses alimentos ou substâncias são chamados de termogênicos.

Outra característica marcante desses alimentos é o maior nível de dificuldade de digestão, ocasionando um maior consumo de energia e caloria quando comparado com outros alimentos. A cafeína, canela, chá de hibisco, gengibre, pimenta vermelha, chá verde, óleo de coco, vinagre de maça são alguns exemplos de alimentos termogênicos que podem ajudar no emagrecimento quando associados com uma dieta balanceada e prática de exercícios físicos (MUNDO BOA FORMA, 2016). Os alimentos citados anteriormente podem ser consumidos de diferentes maneiras, processados e utilizados como complementos de outros alimentos.

Como mencionado, a pimenta malagueta é considerada um alimento termogênico, possuindo um componente ativo chamado capsaicina, responsável por seu sabor picante, sendo esta pungência sua principal característica. O ativo capsaicina é acumulado pelas plantas no tecido da superfície da placenta, sendo liberado pelo dano físico às células quando se extraem sementes ou corta-se o fruto (SANTOS et. al., 2008).

Dentro deste contexto, este trabalho tem como objetivo a avaliação do processo de desidratação osmótica da pimenta malagueta (Capsicum frutescens) variedade malaguetinha.

\section{MATERIAIS E MÉTODOS}

O presente trabalho foi realizado nos Laboratórios de Pesquisas Agropecuárias (LPA) e Laboratório de Cinética e Modelagem de Processos (LACIMP), da Universidade Federal do Tocantins (UFT), Campus Palmas-Tocantins.

A matéria-prima utilizada neste estudo foi a pimenta malagueta, vulgarmente conhecida como maleguitinha in natura, adquirida através de produtores locais dos municípios, Miracema do Tocantins e 
Palmas, ambas cidades tocantinenses. Foram selecionadas pimentas em estádio de maturação comercial completa.

Para a desidratação osmótica, foram pesadas $10 \mathrm{~g}$ das amostras de pimenta in natura inteiras e sem a retirada das sementes e colocada em um béquer contendo a solução desidratante nas concentrações e temperaturas apresentadas na tabela 1 e tampado com plástico filme. Colocou-se em um banho-maria metabólico, onde permaneceu em repouso por 30 minutos. Logo ligou-se a agitação que, no equipamento, vai de uma escala de 0 a 10 sendo a agitação utilizada de 8,5 constantemente. O equipamento apresenta as agitações em escala não identificando a velocidade em cada escala a agitação escolhida foi feita utilizando o critério de movimentar a amostra sem que a solução fosse projetada para fora do recipiente.

Tabela 1 - Variáveis independentes codificadas.

\begin{tabular}{cccccc}
\hline Variáveis & $\mathbf{- \alpha}$ & $\mathbf{- 1}$ & $\mathbf{0}$ & $\mathbf{+ 1}$ & $+\boldsymbol{\alpha}$ \\
\hline Temperatura $\left({ }^{\mathbf{0}} \mathbf{C}\right)$ & 38 & 40 & 45 & 50 & 52 \\
\hline $\begin{array}{c}\text { Concentração da } \\
\text { solução }(\% \text { de } \\
\text { sacarose) }\end{array}$ & 26 & 30 & 40 & 50 & 54 \\
\hline
\end{tabular}

Ao fim da operação de desidratação osmótica, as pimentas foram retiradas da solução, drenadas e secadas superficialmente com papel absorvente. Pesaram-se as amostras em balança analítica, precisão de 0,0001 , para cálculo do conteúdo de umidade.

Os valores obtidos nas determinações analíticas durante a desidratação osmótica foram utilizados para calcular os percentuais de perda de água (PA), ganho de sólidos (GS) do fruto, conforme as equações citadas por Raji et. al. (2010) apresentadas abaixo:

$$
\begin{aligned}
P A & =\frac{(M 0-m o)-(M-m)}{M o} \\
\mathrm{GS} & =\frac{m-m o}{M o}
\end{aligned}
$$

Em que:

$M_{0}$ - Massa inicial antes da desidratação

$M$ - Massa de pimenta após o tempo (t) de desidratação osmótica. $m_{0}-$ Massa de pimenta.

$m$ - Massa seca de pimenta no tempo (t) de desidratação osmótica.

Para a utilização da concentração com chá de canela, utilizou-se 3 palitos de canela de aproximadamente $5 \mathrm{~cm}$ de comprimento para um litro de água destilada. $\mathrm{O}$ recipiente foi colocado em uma chapa de aquecimento para procedimento de cocção. Após o líquido levantar fervura, deixou-se assim por 5 minutos e a chapa foi desligada. Por fim, deixou-se o líquido esfriar até atingir temperatura ambiente. Para a preparação da solução desidratante misturou-se concentrações diferentes de açúcar para as variadas concentrações.

O conteúdo de umidade foi determinado pelo método do Instituto Adolfo Lutz (2008).

\section{RESULTADOS E DISCUSSÃO}

A análise de umidade da pimenta in natura apresentou o valor de $63,37 \%$ o que está de acordo com a literatura, visto que Lutz e Freitas (2008) encontraram valores semelhantes trabalhando com $\mathrm{o}$ valor nutricional de diferentes pimentas consumidas no Brasil. O valor do teor de umidade que eles encontraram para a pimenta malagueta foi de $63,50 \%$.

\section{Desidratação Osmótica}

Os resultados observados foram analisados de acordo com perda de água e ganho de sólidos.

Para a determinação de perda de água observou-se que as variáveis não influenciaram a resposta, não apresentando efeito significativo nem para temperatura nem para a concentração e suas interações, contudo observando o Gráfico de Pareto (Figura 1a) da variável utilizando solução binária de água e açúcar vê-se que a concentração apresenta um valor positivo, indicando que um aumento na concentração pode influenciar a perda de água.

O Gráfico de Pareto (Figura 1b) demonstra que a interação entre as variáveis tem valor negativo, indicando que o aumento das variáveis produz efeito contrário na resposta. Esse comportamento pode estar associado à formação de uma película na superfície da amostra que impede o fluxo de água da amostra para a solução. 


\section{Ganho de Sólidos}

Novamente pode-se observar que não houve efeitos significativos para o ganho de sólidos utilizando solução binária de água e açúcar, contudo o Gráfico de Pareto (Figura 2a) indica que a concentração apresenta valor positivo, indicando que a resposta sofre um aumento à medida que se eleva a concentração da solução (de 26 para $54 \%$ de açúcar).

Figura 1 - Gráfico de Pareto da variável Perda de Água utilizando solução binária de água e açúcar (a) e solução binária de chá de canela e açúcar (b).

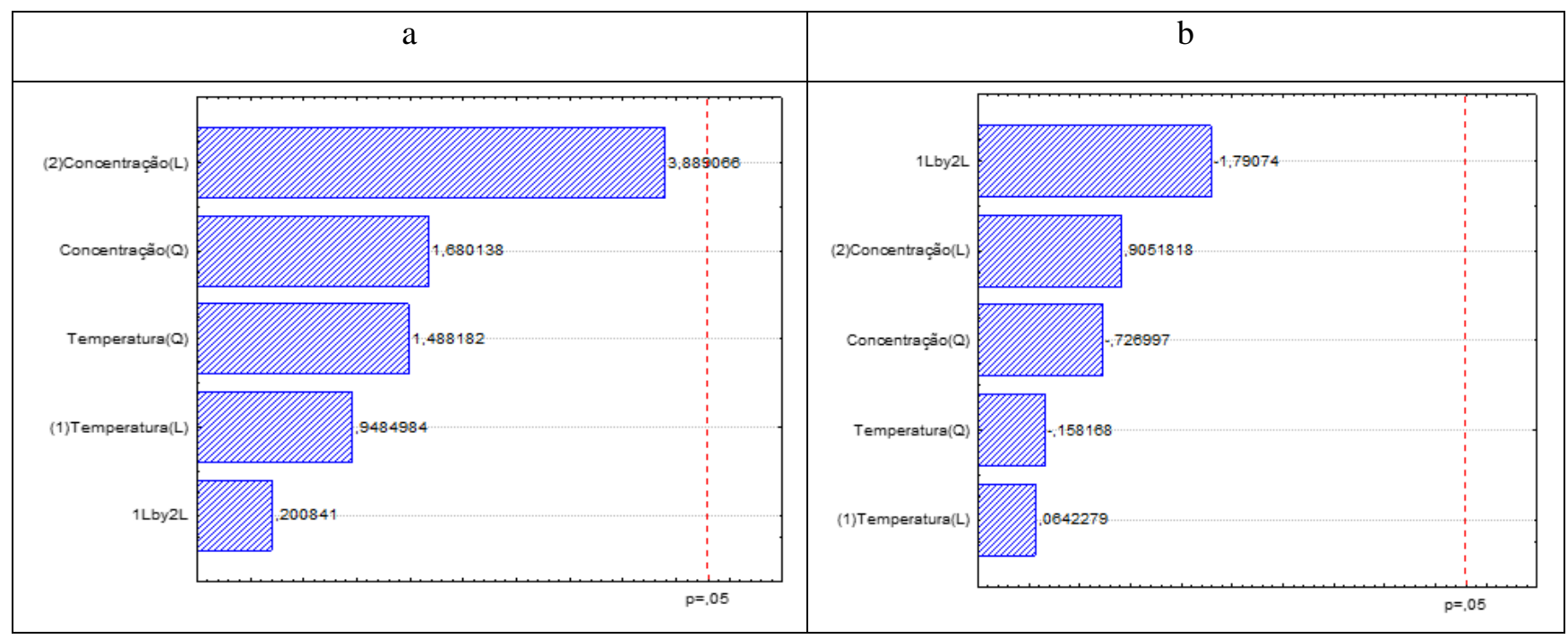

Figura 2 - Gráfico de Pareto da variável Ganho de Sólidos utilizando solução binária de água e açúcar (a) e solução binária de chá de canela e açúcar (b).

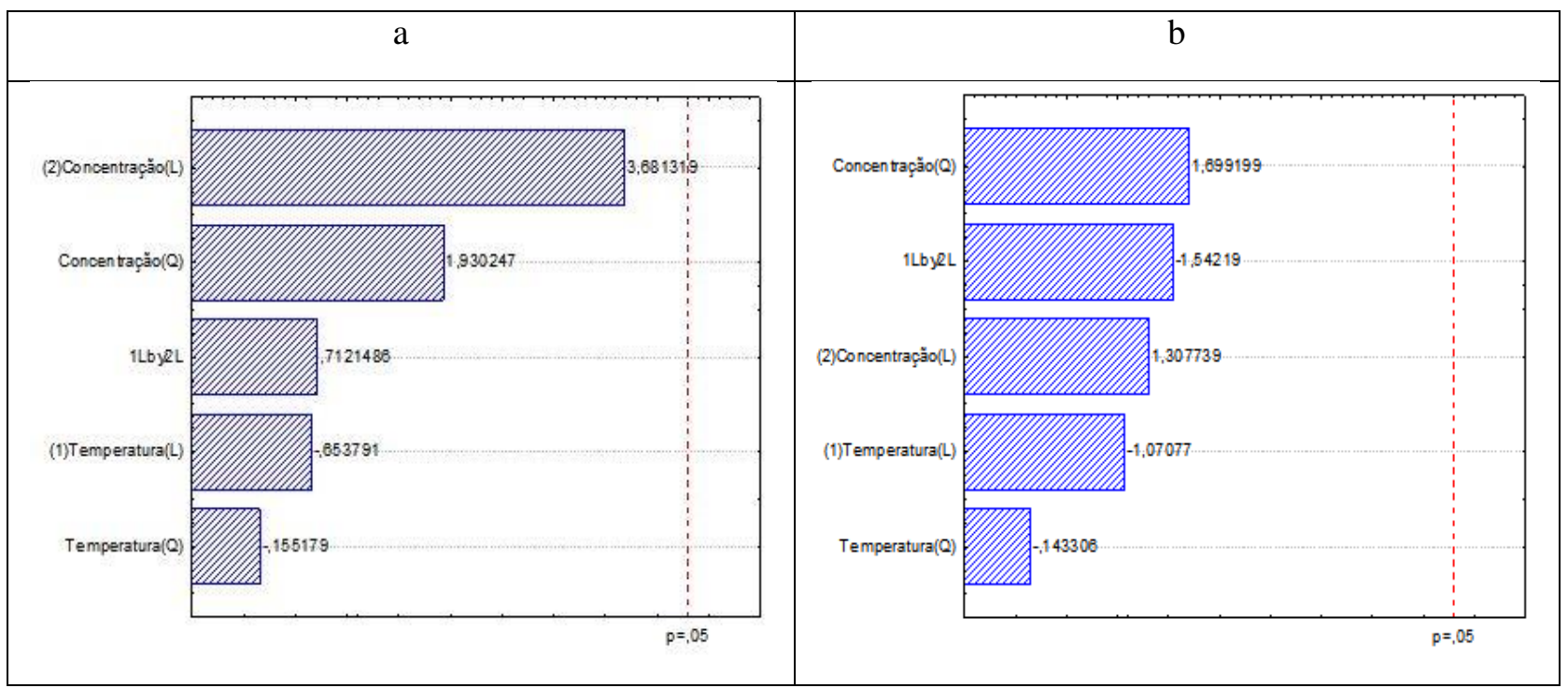

A concentração apresenta valor positivo quando se observa o Gráfico de Pareto (Figura 2b).
Esse comportamento pode indicar uma tendência de que a solução desidratante utilizando chá de canela e 
açúcar com maiores concentrações poderia promover um maior ganho de sólidos na amostra.

As respostas (PA, GS) não obtiveram efeitos significativos na faixa de temperatura e concentração utilizadas, mesmo quando houve a troca do solvente. Como observado na literatura a pimenta apresenta um teor de umidade mais baixo do que o observado para outros vegetais, apresenta ainda valores de teor de lipídeos e fibras mais altos do que os valores para outros tipos de pimentas. Esse comportamento pode ter influenciado as respostas, indicando que mais estudos avaliando outros componentes devam ser considerados para determinar melhores condições para o processo de desidratação de pimenta (Lutz e Freitas, 2008 apud Pinto, 2013).

\section{CONCLUSÃO}

Não houve efeito significativo das variáveis temperatura e concentração na resposta de Perda de Água, contudo a concentração apresenta valor positivo, indicando que essa variável promove a Perda de Água;

A mudança de solvente não influenciou a Perda de Água e as interações entre as variáveis apresenta valor negativo, mostrando tendência à diminuição na resposta ao se aumentar as variáveis;

O Ganho de Sólidos também não apresentou efeito significativo das variáveis, mas a concentração apresenta uma tendência a aumentar a resposta em valores maiores da variável. O mesmo comportamento foi observado na mudança do solvente;

Concluiu-se, portanto, que a faixa de temperatura e concentração não foi eficiente para otimizar o processo e que talvez estudos mais detalhados sobre o teor de umidade, lipídeos e quantidade de fibras associados com essas variáveis devam ser considerados.

Todos os autores declararam não haver qualquer potencial conflito de interesses referente a este artigo.

\section{REFERÊNCIAS}

BIANCO, A. C. Hormônios Tireóides, UCPs e Termogênese. Arquivos Brasileiros de Endocrinologia e Metabolismo, vol. 44, n. 4, São Paulo, 2000. Disponível em: $<$ http://www.scielo.br/scielo.php?script=sci_arttext\& pid=S0004-
$7302000000400003 \& \operatorname{lng}=\mathrm{en} \& \mathrm{nrm}=\mathrm{iso} \& \mathrm{t} \operatorname{lng}=\mathrm{pt}>$. Acessado em: 5 de outubro de 2016.

CASTRO, D. S; NUNES, J. S; JÚNIOR, A. F. S; AIRES, J. E. F; SILVA, W. P; GOMES, J. P. Influência da Temperatura no Processo de Desidratação Osmótica de Pedaços de Goiaba. Revista Geintec - Gestão Inovação e Tecnologias, v. 4, p. 1413-1423, 2014. Disponível em: $<$ http://revistageintec.net/portal/index.php/revista/arti cle/view/405>. Acessado em: 5 de outubro de 2016.

INSTITUTO ADOLFO LUTZ. Métodos físioquímicos para análise de alimentos. Coordenadores Odair Zenebon, NeusSadoccoPascuet e Paulo Tiglea São Paulo: Instituto Adolfo Lutz, p. 1020, 2008. Disponível em: <http://www.crq4.org.br/sms/files/file/analisedealime ntosial_2008.pdf $>$. Acessado em: 15 de agosto de 2016.

LUTZ, D.L.; FREITAS, S.C. Valor Nutricional. In: RIBEIRO, C.S. da C. et al. (Ed.). Pimentas Capsicum. Brasília: Embrapa Hortaliças, 2008, cap.4, p.31-38.

\section{MUNDO BOA FORMA. 10 Melhores Alimentos Termogênicos Naturais para Emagrecer. Disponível em:} $<$ http://www.mundoboaforma.com.br/10-melhoresalimentos-termogenicos-naturais-para-emagrecer/> . Acessado em: 15 de setembro de 2016.

RAJI, A.; FALADE K. O.; BIMBOLU, F. Effect of sucrose and binary solution on osmotic dehydration of pepper varieties (Capsicum spp.) J Food Sci Technol. 2010;47(3):305-309. doi: 10.1007/s13197-010-00487.

SANJINEZ-ARGADONA, E. J.; MENEGALLI, F. C.; HUBINGER, M. D. Desidratação osmótica de goiabas: influência das variáveis do processo na cinética de transferência de massa. Revista Ciências Exatas e Naturais, v. 6, n. 2, p. 203-216, 2004. Disponível em: <http://revistas.unicentro.br/index.php/RECEN/articl e/view/397/548>. Acessado em: 15 de agosto de 2016.

SANTOS, J. A. B.; Silva G. F.; Oliveira L. C. Avaliação dos Capsaicinóides em Pimentas Malagueta. Revista Eletrônica da FJAV. Ano I, no 2, ISSN 1983-1285, 2008. Disponível em: <http://fjav.com.br/revista/Downloads/141_080602_ No02_EdicaocomISSN_.pdf>. Acessado em: 22 de setembro de 2016. 